\title{
Knowledge-Based Acquisition of Tradeoff Preferences for Negotiating Agents*
}

\author{
Xudong Luo, Nicholas R. Jennings, and Nigel Shadbolt \\ Department of Electronics and Computer Science, University of Southampton \\ Southampton SO17 1BJ, United Kingdom. \\ $\{x l, n r j, n r s\} @ e c s . s o t o n . a c . u k$
}

\begin{abstract}
A wide range of algorithms have been developed for various types of automated negotiation. In developing such algorithms the main focus has been on their efficiency and their effectiveness. However, this is only part of the picture. Agents typically negotiate on behalf of their owners and for this to be effective the agent must be able to adequately represent the owners' preferences. However, the process by which such knowledge is acquired is typically left unspecified. To remove this shortcoming, we present a case study indicating how the knowledge for a particular negotiation algorithm can be acquired. More precisely, according to the analysis on the automated negotiation model, we identified that user trade-off preferences play a fundamental role in negotiation in general. This topic has been addressed little in the research area of user preference elicitation for general decision making problems as well. In a previous paper, we proposed an exhaustive method to acquire user trade-off preferences. In this paper, we developed another method to remove the limitation of the high user workload of the exhaustive method. Although we cannot say that it can exactly capture user trade-off preferences, it models the main commonalities of trade-off relations and reflects users' individualities as well.
\end{abstract}

Keyword: tradeoff, negotiation, preference elicitation, decision making software agent, e-Commerce.

\section{INTRODUCTION}

Negotiation - the process by which a group of agents come to a mutually acceptable agreement on some matter - is a key form of interaction in e-commerce systems [16]. Given its importance, a wide range of models have been developed; these include models for auctions, direct one-to-one negotiations, and argumentation-based encounters (see [19] for an overview). To date, however, research in this field has been almost exclusively concerned with the development of efficient and effective algorithms that enable agents to be

*The work is supported by Hewlett Packard Research Labs.

The Fifth International Conference on Electronic Commerce 2003 Pittsburgh, USA successful and obtain acceptable outcomes. While this is clearly important, it is only part of the picture. In most cases, agents negotiate on behalf of their owner (which may be an individual or an organisation). For this to be effective, agents must be able to adequately reflect their owners' interests, preferences, and prejudices in the given domain such that they can negotiate faithfully on their behalf. So, the acquisition/learning of this sort of knowledge can be regarded as an essential aspect of negotiating agent design. However, at this time, little thought has been given to the problems of exactly what knowledge an owner needs to impart to their agent in order to achieve high fidelity negotiation behaviour, and how such knowledge can be effectively acquired from the owner. These are clearly serious shortcomings of existing research that need to be addressed if negotiating agents are to be widely used.

Against this background, our research seeks to start bridging the knowledge acquisition gap between the negotiating agents' owners and the negotiation algorithms that their agents use. In this paper, we focus on exploring how the necessary knowledge of tradeoff preferences can be acquired. Here term tradeoff among negotiation issues refers to a set of combinations of the issues' values in which making some issues' values worse can be balanced by improving other issues' values (elements of the set are called tradeoff alternative), and term tradeoff preference refers to an ordering on a set of tradeoff alternatives (this is realised through assigning each alternative a satisfaction degree). Tradeoff is a fundamental aspect in the principled negotiation approach and, in general, is necessary in many other negotiation models (e.g., [7]). This is because negotiating participants are usually assumed to be their interest maximisers and a tradeoff alternative can minimise the loss of their interest but may increase the interest of their negotiation partners (thus win-win solutions could be reached). So, although our exploration is carried out for a particular negotiation scenario [21], its method for eliciting tradeoff preference is significant for negotiation in general. Also, it contributes towards extending the research area of user preference elicitation where the problem has been addressed little.

In the previous paper [20], we develop an exhaustive acquisition method to obtain user tradeoff preferences. It works in this way: first make some attributes worse, then ask users to adjust other attributes to get a balance, and finally asks the users to assign satisfaction degree to each tradeoff alternative. According to standard business negotiation theory [1, 
$8,25,26,30]$, before doing a real negotiation, people should explore options as widely as they can. So, when our system makes some attributes worse, it explores options as widely as possible. This method is good for acquiring trade-offs between two attributes that are the most common. However, its workload for users in eliciting tradeoff preferences values is very high because: (1) it exhaustively explores the whole space of all possible tradeoff alternatives; and (2) it assumes that the human users must be aware of the attributes between which the tradeoffs exist and the types of tradeoffs. This motivates the further work that aims at reducing the workload (most heuristic methods for eliciting user preferences $[12,14,13,10,27,15,6]$ have similar motivation). Thus, like that used in heuristic methods of user preferences elicitation, neural networks, case based reasoning and inductive learning methods are alternative techniques for reducing the elicitation workload of user tradeoff preference. Nevertheless, these alternative techniques assume that a lot of data are available. This is true in many domains, but not always in this case especially for a new user. Thus, in this paper we chose to design a meta-knowledge based method for acquiring user tradeoff preference. Its advantage over these potential alternative techniques ${ }^{1}$ is that it does not require a large amounts of user data before it works (i.e., plug in and play). However, like all similar heuristic method, the accuracy of the method proposed in this paper is lower than the exhaustive method although the one here is more efficient.

Additionally, our work is also significant at the following two aspects. Firstly, the problem of user tradeoff preference acquisition has been addressed little in the research areas of general knowledge acquisition and preference elicitation. Accordingly, our work can be viewed as a contribution to these areas. Secondly, to address the problems of knowledge acquisition for other types of negotiation models is less significant. The decision models of most negotiation systems are utility-based, reasoning-based and constraintbased. The utility acquisition has been revisited and refined over the past 50 years, and the main results of this research effort are now well established. so, even if we applied it to automated negotiation, little could make it special (e.g., [11]). As to reasoning based models, knowledge used for reasonings is mostly modelled as Bayesian networks, fuzzy rules, or cases; there also exist well-established methods for acquiring these forms of knowledge (either from data or from human users). Even if we applied these existing methods to acquire knowledge for reasoning-based negotiation systems, the contribution would be limited since we just applied the existing methods. Nevertheless, there are no general methods for constraint acquisition and thus we have to invent some new methods ourselves to solve the problem that has not been solved yet.

\footnotetext{
${ }^{1}$ However, the alternatives are worth studying since generally speaking different approaches are appropriate for different classes of applications. Actually, besides presenting the first attempts on the topic of tradeoff preference elicitation, another more important aim of this paper is to motivate more work on this topic and, more generally, to develop a thorough understanding of the opportunities and challenges, problems and candidate solutions, that arise in acquiring sufficient user requirements and preferences to build software agents that are able to negotiate effectively.
}

The remainder of this paper is structured as follows. Section 2 presents our knowledge-based model for acquiring user tradeoffs. Sections 3-5 identify three common types of tradeoff relations. Section 6 presents knowledge that is used in our knowledge-based acquisition system for generating concrete tradeoff relations. Section 7 discusses related work. Finally, Section 8 concludes and outlines the avenues of further research.

\section{KNOWLEDGE-BASED ACQUISITION}

This section outlines our knowledge based system of tradeoff acquisition. Fig. 1 shows the basic idea behind the method. First, the system queries the user about choice features in order to determine which attributes the trade-off relations exist between. Second, in oder to determine the shape of the tradeoff curve, the system queries the user about the relative importance degree of one attribute against another and about some features of tradeoff curves. Finally, the system queries the user about his satisfaction degree for each tradeoff alternative. For example, in the accommodation renting scenario (described in [21]) a user consults the system how to reduce rental rate via tradeoff. First, the system could query him about his choice: e.g., take accommodation that is further away from the desired location or for a longer rental period? If the user tells the system that he likes to take it further away, the system will suggest the tradeoff is between distance and rate. Then the system queries about which attributes, rate or distance, is more important. In the case the user rates the distance as more important, the system will further query the user about some features that are useful in determining the curve shape of tradeoff between distance and rate. For instance, the system could ask the user whether medium distance is important. If the user's answer is negative, the system will advice the tradeoff takes the curve of adaptive tradeoff relation (details on this it later). Finally, the system asks the system to assign satisfaction degrees to the tradeoff alternatives.

The system consists of a number of knowledge bases and inference functions that we will explain in the following.

The knowledge bases that are applied in the process of tradeoff acquisition are choice rules and generation rules. A choice rule is used to choose the attributes a tradeoff relation exists between. The condition part of a choice rule consists of some choice features that the system will ask a user to confirm, and its conclusion part specifies which attributes the trade-off exists. We can have such rules because various attributes that tradeoffs exist between have been identified by human negotiation theory [29, 30, 31]. For example, in business negotiation, usually tradeoffs exist between price and quality, between price and quantity, and so on. In our accommodation renting scenario [21], we consider two kinds of tradeoff relations: (1) the tradeoff between rental rate and rental period, and (2) the tradeoff between rental rate and walking distance. In this scenario, choice rules are used to choose a tradeoff relations from these two. For example, in the scenario, we have choice rules like "IF moving='like'^ busy='yes' THEN use 'tradeoff between rent and period". .'

\footnotetext{
${ }^{2}$ This rule means that if the potential tenant likes to move from one place to another so that he can often make more friends and if he is too busy to spent much time on the journey then he can choose longer rental period to trade off
} 


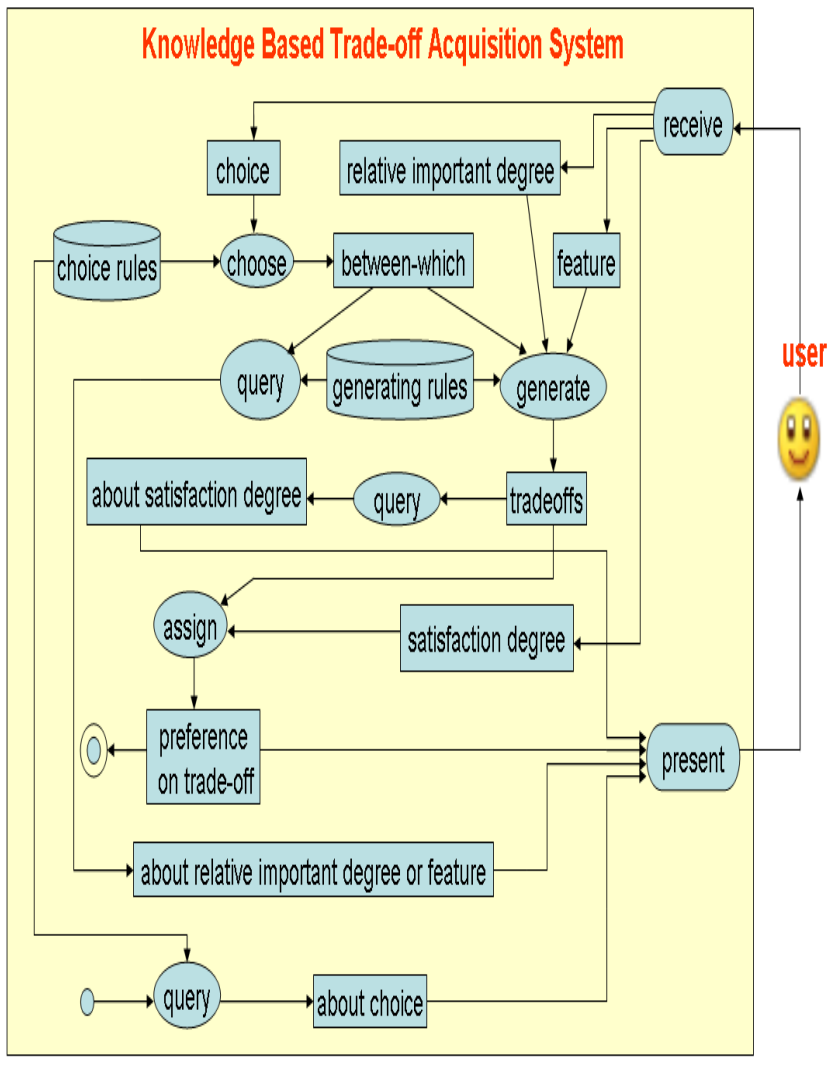

Figure 1: Graphical specification of the task model of the tradeoff acquisition system. The following graphical conventions are used: Rectangles represent dynamic knowledge roles. The name of the knowledge role is written in the rectangle. Ovals represent inferences. Arrows are used to indicate input-output dependencies between roles and inferences. The name of a static knowledge role is written in a cylinder. The bullet represents the start point and the o-bullet represents the end point.

After determining which attributes a tradeoff relation exists between, the generation rules are used to determine the specific shape of the tradeoff relation. In this paper, we identified three common types of tradeoff relations. We shall discuss these in detail in the next three sections. While discussing these three types of tradeoff relations, according to principles of software and knowledge engineering [28], we first present their axiom framework and then instantiate these axioms. Actually, the axioms identified define the requirements of some common tradeoffs, and then in order to fit these requirements we instantiate them.

The following inferences and two transfer functions are employed in the process of tradeoff acquisition: (1) query: The inference function specifies the question about information that the system needs in order to determine tradeoff relations. The information includes the choice information of attributes that tradeoffs exist between, relative degree of

the rental rate. importance of one attribute against another, and features of various tradeoff types. (2) choose: The inference function chooses attributes that a tradeoff should be made between. (3) generate: According to the relative degree of importance of one attribute against another as well as the feature information of tradeoff types, the inference function determines the concrete curve of the tradeoff relation. (4) assign: The inference allows the user to assign and adjust their satisfaction degrees to tradeoff alternatives that have been identified. (5) present: This transfer function presents information (questions, tradeoff alternatives, and preference on tradeoff alternatives) to the user. (6) receive: This transfer function reads in information (the answer to the question, the relative importance degree of one attribute against another, and the satisfaction degrees of tradeoff alternatives).

\section{THE QUALITY TRADEOFF}

This section discusses a kind of tradeoff relation where the concession of good value of one attribute is used to improve the $b a d$ value of another attribute, and thus we call it a quality tradeoff. The basic idea of this kind of tradeoff between attributes $x$ and $y$ is inspired by the following intuitions that people make tradeoffs between two attributes:

1. If attribute $x$ is more important than attribute $y$, then when $y$ 's good values ${ }^{3}$ are used to tradeoff $x$ 's very bad values, the user is willing to make $y$ a lot worse to get $x$ a little better; and the worse $x$ is the more one needs to make $y$ worse. For example, in our accommodation renting scenario, if the rental rate is more important than the walking distance, then when the rental rate is $£ 330$ and the walking distance is (almost) no time, the user might be willing to increase walking distance to 15 minutes (half to the worst value, or say $50 \%$ worse) to get the rental rate reduced to $£ 250$ (about $24 \%$ better). This is because each month he saves $330-250=80$ pounds; so if he needs to go to university once each day, it means that he makes $\frac{80}{30}=2.67$ pounds by spending $15+15=30$ minutes-clearly it is worth if it is regarded as a kind of part time job (suppose that for a student the minimum payment of a part time job is 5 pounds per hour).

2. If attribute $x$ is less important than attribute $y$, then when $y$ 's good values are used to tradeoff $x$ 's very bad values, the user is willing to make $y$ a little worse in order to get $x$ a lot better and the better $y$ 's value is the less one needs to make $y$ worse in order to get $x$ better. For example, in the accommodation renting scenario, if the rental rate is less important than the walking distance, then when the rental rate is $£ 330$ and the walking distance takes (almost) no time, the user is willing to increase walking distance to $10 \mathrm{~min}-$ utes (about 33\% worse) for reducing the rental rate to $£ 150$ (about $54 \%$ better).

3. If attribute $x$ is equally important as attribute $y$, then the user is willing to make $y$ worse to the same extent of

\footnotetext{
${ }^{3}$ Generally speaking, it is a fuzzy concept. However, in this paper we regard a value as a good value if it is greater than a threshold. Regarding it as a fuzzy concept falls outside the scope of the current paper although it is certainly a subject of further research.
} 
getting $x$ better. Again, in the accommodation renting scenario, if the rental rate is equally important as the walking distance, then when the rental rate is $£ 330$ and the walking distance is (almost) no time, the user is willing to increase walking distance to 16 minutes (about $53 \%$ worse) for reducing the rental rate to $£ 154$ (about $53 \%$ better).

Formally, let the range of attribute $x$ be $[a, b]$ and the range of attribute $y$ be $[c, d]$, where $a, c \geq 0$. Suppose the user thinks the greater $x$ 's value is the worse it is, and the greater $y$ 's value is the worse it is. Thus, the alternative tradeoffs between $x$ and $y$ can be defined as a relation $f:[a, b] \rightarrow$ $[c, d]$. Then, the first intuition means that when $x$ is not smaller than a point $x_{0}$ the decrement rate of $x$ is smaller than the increment rate of $y=f(x)$, i.e.,

$$
x_{0} \leq x_{1} \leq x_{2} \Rightarrow \frac{x_{2}-x_{1}}{b-a}<\frac{f\left(x_{1}\right)-f\left(x_{2}\right)}{d-c} ;
$$

the second intuition means that when $x$ is not smaller than a point the decrement rate of $x$ is greater than the increment rate of $y=f(x)$, i.e.,

$$
x_{0} \leq x_{1} \leq x_{2} \Rightarrow \frac{x_{2}-x_{1}}{b-a}>\frac{f\left(x_{1}\right)-f\left(x_{2}\right)}{d-c} ;
$$

and the third intuition means that the decrement rate of $x$ is the same as the increment rate of $y=f(x)$, i.e.,

$$
x_{0} \leq x_{1} \leq x_{2} \Rightarrow \frac{x_{2}-x_{1}}{b-a}=\frac{f\left(x_{1}\right)-f\left(x_{2}\right)}{d-c} .
$$

And with respect to (1), we have:

$$
\frac{f\left(x_{2}\right)-f\left(x_{1}\right)}{x_{2}-x_{1}}<-\frac{d-c}{b-a} \Rightarrow \lim _{x_{2}-x_{1} \rightarrow 0} \frac{f\left(x_{2}\right)-f\left(x_{1}\right)}{x_{2}-x_{1}}<-\frac{d-c}{b-a} ;
$$

with respect to $(2)$, we have:

$$
\frac{f\left(x_{2}\right)-f\left(x_{1}\right)}{x_{2}-x_{1}}>-\frac{d-c}{b-a} \Rightarrow \lim _{x_{2}-x_{1} \rightarrow 0} \frac{f\left(x_{2}\right)-f\left(x_{1}\right)}{x_{2}-x_{1}}>-\frac{d-c}{b-a} ;
$$

and with respect to (3), we have:

$$
\frac{f\left(x_{2}\right)-f\left(x_{1}\right)}{x_{2}-x_{1}}=-\frac{d-c}{b-a} \Rightarrow \lim _{x_{2}-x_{1} \rightarrow 0} \frac{f\left(x_{2}\right)-f\left(x_{1}\right)}{x_{2}-x_{1}}=-\frac{d-c}{b-a} .
$$

In mathematical calculus,

$$
\lim _{x_{2}-x_{1} \rightarrow 0} \frac{f\left(x_{2}\right)-f\left(x_{1}\right)}{x_{2}-x_{1}}
$$

is the derivative of $y=f(x)$, denoted as $y^{\prime}$.

Moreover, intuitively when the degree, $\alpha$, to which attribute $x$ is more or less important than attribute $y$ is different, the tradeoff relations between them should be different. More specifically,

- when $x$ is more important than $y$, the greater $\alpha$ the more concession at $y$ is needed in order to get $x$ better a little from its worst value;

- when $x$ is less important than $y$, the smaller $\alpha$ the less concession at $y$ from its best value is needed in order to get $x$ better a lot; and
- when $\alpha$ is approaching to 0.5 (i.e., $x$ is equally important as $y$ ), the rate of the concession at $y$ is approaching to the rate of the improvement at $x$.

We let this kind of degree take its value on $[0,1]$ :

- In the case $\alpha \in(0.5,1]$, the greater $\alpha$ the more $x$ is more important than $y$ (in particular, when $\alpha$ is $1, x$ is completely more important than $y$ ). So, the greater $\alpha$ the more concession at $y$ is needed to get $x$ better from its worse value.

- In the case $\alpha \in[0,0.5)$, the smaller $\alpha$ the less $x$ is more unimportant than $y$ (in particular, when $\theta$ is 0 , $x$ is completely unimportant compared with $y$ ).

Thus, formally we have the following definition:

Definition 1. Suppose $\alpha \in[0,1]$ is a degree to which attribute $x$ is more or less important than attribute $y$. For a given value of $\alpha$, let $f_{\alpha}:[a, b] \rightarrow[c, d]$ be not increasing, and $\forall \alpha \in[0,1], f_{\alpha}(a)=d, f_{\alpha}(b)=c$. Then $y=f_{\alpha}(x)$ is a quality tradeoff relation between $x$ and $y$ if there is a constant $x_{0} \in[a, b]$ such that

1. when $x$ is more important than $y$, the following properties hold:
(a) $x \geq x_{0} \Rightarrow y^{\prime}<-\frac{d-c}{b-a}$
(b) $x_{1} \geq x_{2} \Rightarrow y_{1}^{\prime} \geq y_{2}^{\prime}$,
(c) $\alpha_{1} \leq \alpha_{2} \Rightarrow \frac{f_{\alpha_{1}}(x)-c}{b-x} \leq \frac{f_{\alpha_{2}}(x)-c}{b-x}$, and
(d) $\lim _{\alpha \rightarrow 0.5} y=d-\frac{d-c}{b-a}(x-a)$;

2. when $x$ is less important than $y$, the following properties hold:
(a) $x \geq x_{0} \Rightarrow y^{\prime}>-\frac{d-c}{b-a}$
(b) $x_{1} \leq x_{2} \Rightarrow y_{1}^{\prime} \geq y_{2}^{\prime}$,
(c) $\alpha_{1} \leq \alpha_{2} \Rightarrow \frac{f_{\alpha_{1}}(x)-c}{b-x} \leq \frac{f_{\alpha_{2}}(x)-c}{b-x}$, and
(d) $\lim _{\alpha \rightarrow 0.5} y=d-\frac{d-c}{b-a}(x-a)$; and

3. when $x$ is equally important as $y, y^{\prime}=-\frac{d-c}{b-a}$.

The above definition lists the axioms that formally represent human tradeoff commonalities we discussed above. The following theorem gives an instantiation of the generic framework of these axioms.

TheOREM 1. Let $\alpha \in[0,1]$ is a degree to which attribute $x \in[a, b]$ is more important than attribute $y \in[c, d]$. The relation

$$
y= \begin{cases}(d-c)\left(1-\frac{x-a}{b-a}\right)^{2(1-\alpha)}+c & \text { if } \alpha \in(0.5,1] \\ (d-c)\left(1-\left(\frac{x-a}{b-a}\right)^{2 \alpha}\right)+c & \text { if } \alpha \in[0,0.5]\end{cases}
$$

is a quality tradeoff relation between $x$ and $y$. 
The above theorem indeed realises the idea of making $y$ worse to get $x$ better. For example, from the proof of the above theorem, ${ }^{4}$ we can see that:

- In the case that $\alpha=\frac{3}{4}$, when $x$ is improved from $d$ to $\frac{3}{4}(b-a)+a, y$ becomes worse from $c$ to

$$
(d-c) \sqrt{1-\frac{\left(\frac{3}{4}(b-a)+a\right)-a}{b-a}}+c=\frac{d-c}{2}+c .
$$

This means that when $x$ is more important than $y$, the concession of $50 \%\left(\frac{\left(\frac{d-c}{2}+c\right)-c}{d-c}\right)$ of $y$ can get the improvement of $25 \%\left(\frac{b-\left(\frac{3}{4}(b-a)+a\right)}{b-a}\right)$ of $x$.

- In the case that $\alpha=\frac{1}{4}$, when $x$ is improved from $b$ to $\frac{1}{4}(b-a)+a, y$ becomes worse from $c$ to

$$
(d-c)\left(1-\sqrt{\frac{\left(\frac{1}{4}(b-a)+a\right)-a}{b-a}}\right)+c=\frac{d-c}{2}+c .
$$

This means that when $x$ is less important than $y$, the concession of $50 \%\left(\frac{\left(\frac{d-c}{2}+c\right)-c}{d-c}\right)$ of $y$ can get the improvement of $75 \%\left(\frac{b-\left(\frac{1}{4}(b-a)+a\right)}{b-a}\right)$ of $x$.

Now we apply tradeoff relation (7) in our accommodation renting scenario. This involves three attributes rate, period and distance that have domains of $[0,330],[0,12]$ and $[0,30]$, respectively. For the student, the greater the value of rate the worse it is (its worst value $£ 330$ and its best value is $£ 0$ ); the greater the value of period the worse it is (its worst value 12 months and its best value is 0 month); and the greater the value of distance the worse it is (its worst value 30 minutes and its best value is 0 minute). Thus, by (7) the quality tradeoff relation between rate $(r)$ and period $(p)$ are as follows:

$$
p= \begin{cases}12\left(1-\frac{1}{330} r\right)^{2(1-\alpha)} & \text { if } \alpha \in(0.5,1] \\ 12\left(1-\left(\frac{1}{330} r\right)^{2 \alpha}\right) & \text { if } \alpha \in[0,0.5]\end{cases}
$$

and the quality tradeoff relation between rate $(r)$ and distance $(d)$ are as follows

$$
d= \begin{cases}30\left(1-\frac{1}{330} r\right)^{2(1-\alpha)} & \text { if } \alpha \in(0.5,1] \\ 30\left(1-\left(\frac{1}{330} r\right)^{2 \alpha}\right) & \text { if } \alpha \in[0,0.5]\end{cases}
$$

From Fig. 2 of tradeoff relation $(9),{ }^{5}$ it can be seen that:

- When rate is more important than distance the good values of distance are decreased to improve rate, the rate of getting rate better is smaller than that of making distance worse. Intuitively, it means that the user is willing to make distance a lot worse in order to get rate a little better. Moreover, the greater the relative importance degree $\alpha$ the more concession at distance

\footnotetext{
${ }^{4}$ We omitted the proofs of all theorems in this paper due to space limitations.

${ }^{5}$ The figures of tradeoff relation (8) is similar, and so omitted.
}

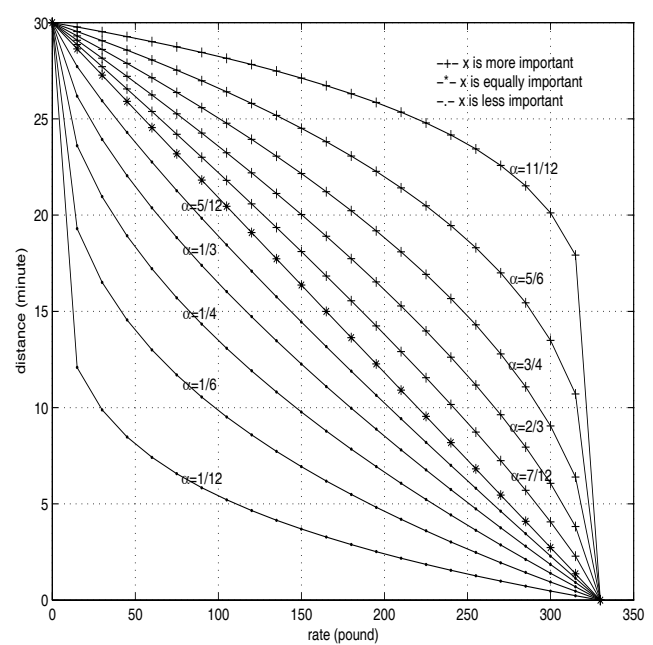

(a) Tradeoff relations between rate and distance for given relative importance degrees.

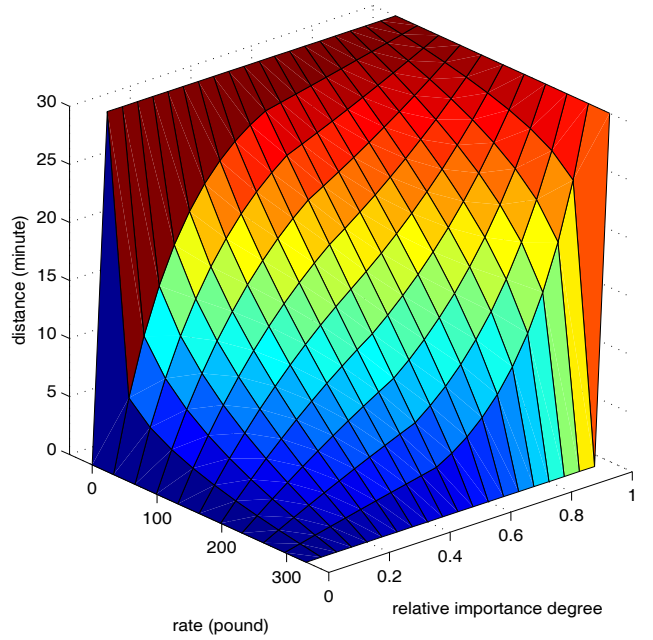

(b) Tradeoff relation between rate and distance for various importance degrees.

Figure 2: The quality tradeoff relations.

is needed in order to get rate better a little from its worst value.

- When rate is less important than distance and the good values of distance are decreased to improve rate, the rate of getting rate better is greater than that of making distance worse. Moreover, the smaller the relative importance degree $\alpha$ the less concession at another attribute from its best value is needed in order to get rate better a lot.

- When rate and distance are equally important, the rate of getting rate getting better is the same as that of making distance worse.

Different users may have different opinion about how much one attribute is more important than another. So, the relative importance of one attribute against another actually reflects a user's individuality (i.e., the user's preference). So, among a family of tradeoff relations (7), for a specific user, 
the one that takes his preference is better than any other. Without considering user preferences, we cannot talk about whether a given tradeoff relation is better than another.

\section{THE IMPASSE TRADEOFFS}

In quality tradeoffs, an attribute is always made worse to get another better. However, this may not be true in all cases. For example, although attribute $y$ is less important than attribute $x$, it may have a perceived bottom line. Tradeoffs may reach an impasse at the bottom line. However, the desirable attribute's value can suddenly become very much attractive so that the users may feel the values of the other attribute can go beyond the bottom line; after that moment, since the value of the more important attribute becomes very good, it is reasonable to make the less important attribute a lot worse to get the more important attribute a little better. The following definition captures this intuition.

Definition 2. Suppose $\alpha \in(0.5,1]$ is a degree to which attribute $x$ is more important than attribute $y$. For a given value of $\alpha$, let $f_{\alpha}:[a, b] \rightarrow[c, d]$ be not increasing, $f_{\alpha}(a)=d$ and $f_{\alpha}(b)=c$. Let $\beta \in[c, d]$ is one constant, called bottom line of $y$. Then $y=f_{\alpha}(x)$ is an impasse tradeoff relation between $x$ and $y$ if $\exists a_{1}, b_{1}, a_{2}, b_{2} \in[0,1]$,

$$
\begin{aligned}
& \forall x \in\left[a_{1}, b_{1}\right], f(x)=\beta, \\
& \forall x \in\left[a, a_{2}\right) \cup\left(b_{2}, b\right], y^{\prime}<-\frac{d-c}{b-a} \\
& \forall x_{1}, x_{2} \in\left[a, a_{2}\right), x_{1} \leq x_{2} \Rightarrow y_{1}^{\prime} \leq y_{2}^{\prime}, \\
& \forall x_{1}, x_{2} \in\left(b_{2}, b\right], x_{1} \leq x_{2} \Rightarrow y_{1}^{\prime} \geq y_{2}^{\prime}, \\
& \forall x \in\left[a, a_{1}\right), \alpha_{1} \geq \alpha_{2} \Rightarrow \frac{d-f_{\alpha_{1}}(x)}{x-a} \geq \frac{d-f_{\alpha_{2}}(x)}{x-a} \\
& \forall x \in\left(b_{2}, b\right], \alpha_{1} \geq \alpha_{2} \Rightarrow \frac{f_{\alpha_{1}}(x)-c}{b-x} \geq \frac{f_{\alpha_{2}}(x)-c}{b-x}
\end{aligned}
$$

In the above definition, (10) means that $\beta$ is the bottom line of $y$ where it is stuck after $x$ is improved from $b_{1}$ to $a_{1} ;(11)$ means that the good value of the less important attribute $y$ is decreased a lot to improve a little the very bad value of the more important attribute $x$, and the bad value of the less important attribute $y$ is decreased a lot to improve a little the very good value of the more important attribute $x$; (12) means that the better the very good value of $x$ the more concession at $y$ needs to be made to get $x$ further better; (13) means that the worse the very bad value of $x$ the more concession at $y$ needs to be made to get $x$ better; (14) means that the more important $x$ is than $y$ the more concession at $y$ is needed to get $\mathrm{x}$ closer a little to its best value; and (15) means that the more important $x$ is than $y$ the more concession at $y$ is needed to get $\mathrm{x}$ better a little from its worst value.

The following theorem gives an impasse tradeoff relation.

Theorem 2. Suppose the domain of attribute $x$ is $[a, b]$ and the domain of attribute $y$ is $[c, d], \alpha \in(0.5,1]$ is a degree to which $x$ is more important than $y$, and $\beta \in[c, d]$ is bottom line of $y$. Then the relation, defined as

$$
y=\left\{\begin{array}{c}
(d-c)\left(1-\frac{x-a}{b-a}\right)^{2(1-\alpha)}+c \\
\quad \text { if } x \in\left(x_{\text {very-bad }}, b\right] \\
\quad \text { if } x \in\left[x_{\text {very-good }}, x_{\text {very-bad }}\right], \\
(d-c)\left(1-\left(\frac{x-a}{b-a}\right)^{2(1-\alpha)}\right)+c \\
\text { if } x \in\left[a, x_{\text {very-good }}\right),
\end{array}\right.
$$

is an impasse tradeoff relation between $x$ and $y$, where

$$
\begin{aligned}
& x_{\text {very-good }}=(b-a)\left(1-\frac{\beta-c}{d-c}\right)^{\frac{1}{2(1-\alpha)}}+a, \\
& x_{\text {very-bad }}=(b-a)\left(1-\left(\frac{\beta-c}{d-c}\right)^{\frac{1}{2(1-\alpha)}}\right)+a .
\end{aligned}
$$

Now we apply the impasse tradeoff relation (16) in our accommodation renting scenario. From (16) the impasse tradeoff relation between between rate $(r)$ and period $(p)$ is:

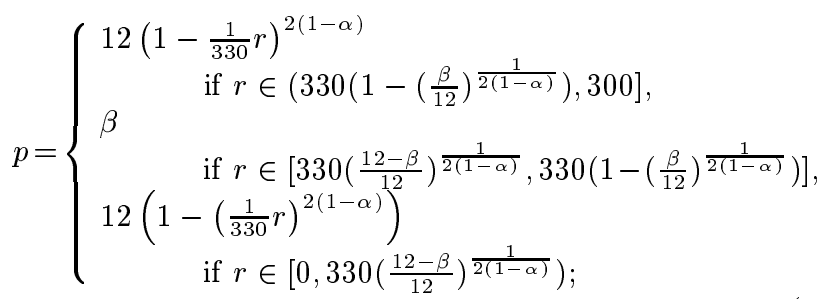

and the impasse tradeoff relation between rate $(r)$ and distance $(d)$ is:

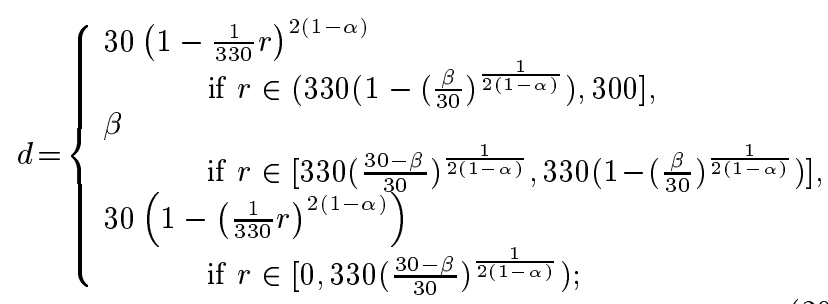

In (19), if a user specifies $\alpha=\frac{1}{4}$ and $\beta=6$, then the impasse tradeoff relation between $\operatorname{rate}(r)$ and $\operatorname{period}(p)$ is

$$
p= \begin{cases}12 \sqrt{1-\frac{1}{330} r} & \text { if } r \in\left(330 \times \frac{3}{4}, 300\right], \\ 6 & \text { if } r \in\left[330 \times \frac{1}{4}, 330 \times \frac{3}{4}\right], \\ 12\left(1-\sqrt{\frac{1}{330} r}\right) & \text { if } r \in\left[0,330 \times \frac{3}{4}\right) ;\end{cases}
$$

and in (20), if a user specifies $\alpha=\frac{1}{4}$ and $\beta=6$, then the impasse tradeoff relation between rate $(r)$ and distance $(d)$ is:

$$
d= \begin{cases}30 \sqrt{1-\frac{1}{330} r} & \text { if } r \in\left(330 \times \frac{3}{4}, 300\right], \\ 15 & \text { if } r \in\left[330\left(330 \times \frac{1}{4} 330 \times \frac{3}{4}\right],\right. \\ 30\left(1-\sqrt{\frac{1}{330} r}\right) & \text { if } r \in\left[0,330 \times \frac{1}{4}\right) .\end{cases}
$$

From the figures (Fig. 3) of these relations, it can be seen that:

- When rate is very bad (i.e., more than $\frac{3}{4} \times 330$ ), since it is more important than another attribute, the user is 


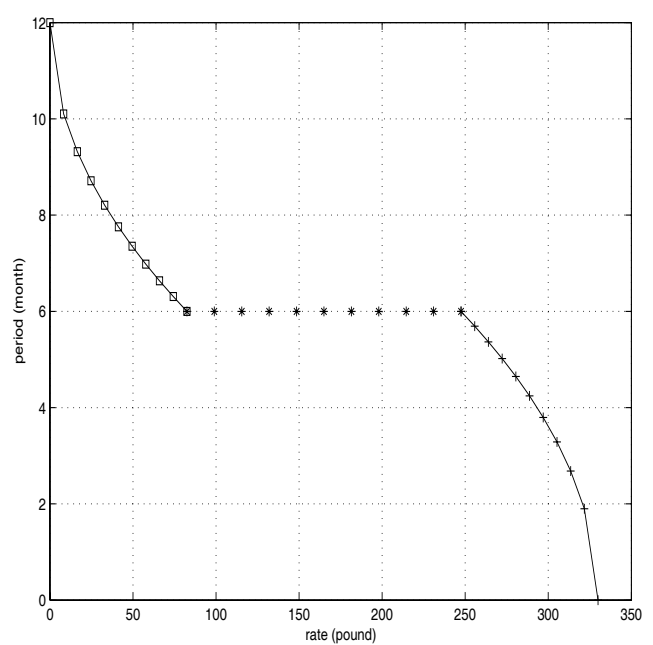

(a) Tradeoff between rate and period.

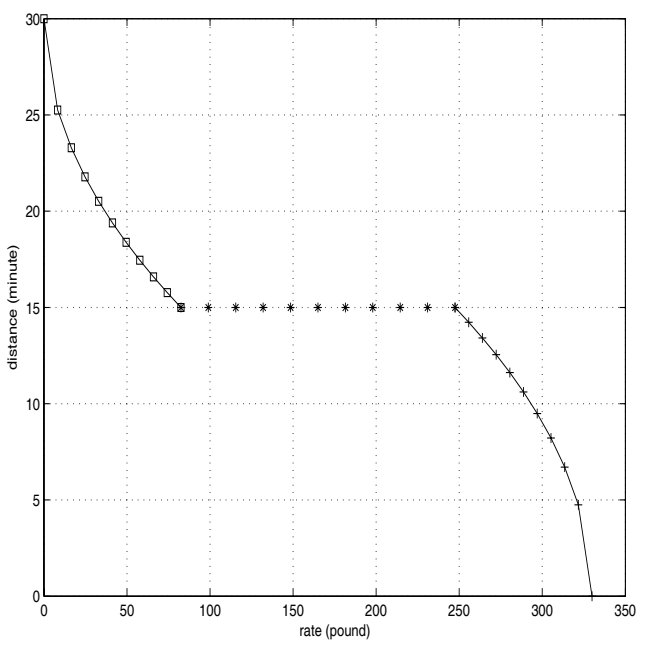

(b) Tradeoff between rate and distance.

Figure 3: The impasse tradeoff relations.

willing to make it a lot worse to get rate a little better; and as the other gets worse the speed of getting rate better becomes less.

- When the other decreases to its bottom line (i.e., 6 months for attribute period, and 15 minutes walking for attribute distance), the user is unwilling to make it worse further unless rate becomes very good (i.e., less than $\left.\frac{1}{4} \times 330\right)$.

- When rate becomes very good and thus very attractive, the user may become interested in bringing rate down further by making the other a lot worse.

For example, for a student who wants to rent an accommodation with limited budget, when the accommodation with good distance is very expensive, he is willing to increase the distance a lot to get accommodation cheaper a little; but when the distance reaches his bottom line he becomes unwilling to increase the distance further until the rental rate becomes very good; after the rental rate becomes very good, he likes to bring it down further by increasing distance a lot since at the moment the rental rate is so attractive for him and the distance is already not good any more.

\section{THE ADAPTIVE TRADEOFF}

In some cases, the negotiator's attitude about which attribute between $x$ and $y$ is more important can change during the course of a tradeoff. For example, (1) when $y$ takes on very good values it can be viewed as more important than $x$, and so making $y$ a little worse should get $x$ a lot better; (2) when $y$ 's value becomes not very good but $x$ 's value is still not very good, the user would like to make $y$ a lot worse to get $x$ a little better, but this cannot go too far; thus (3) when $y$ becomes too bad the user will not make $y$ a lot worse to get $x$ a little better, or say $y$ is viewed more important than $x$ again, and so the user makes $y$ a little worse to get $x$ a lot better. These intuitions can be captured by the following definition.

Definition 3. Suppose $\alpha \in[0,0.5)$ is a degree to which attribute $x$ is more important than attribute $y$. For a given value of $\alpha$, let $f_{\alpha}:[a, b] \rightarrow[c, d]$ be not increasing, $f_{\alpha}(a)=d$ and $f_{\alpha}(b)=c$. Let $f:[a, b] \rightarrow[c, d]$ be decreasing, $f(a)=d$ and $f(b)=c$. Then $y=f(x)$ is an adaptive tradeoff relation between $x$ and $y$ if $\exists a_{1}, b_{1}, a_{2}, b_{2} \in[0,1]$,

$$
\begin{aligned}
& \forall x \in\left[a_{1}, b_{1}\right] \Rightarrow y^{\prime}<-\frac{d-c}{b-a} \\
& \forall x \in\left[a, a_{2}\right) \cup\left(b_{2}, b\right] \Rightarrow y^{\prime}>-\frac{d-c}{b-a} \\
& \forall x_{1}, x_{2} \in\left[a, a_{2}\right), x_{1} \leq x_{2} \Rightarrow y_{1}^{\prime} \geq y_{2}^{\prime} \\
& \forall x_{1}, x_{2} \in\left(b_{2}, b\right], x_{1} \leq x_{2} \Rightarrow y_{1}^{\prime} \geq y_{2}^{\prime} \\
& \forall x \in\left[a, a_{1}\right), \alpha_{1} \leq \alpha_{2} \Rightarrow \frac{d-f_{\alpha_{1}}(x)}{x-a} \leq \frac{d-f_{\alpha_{2}}(x)}{x-a} \\
& \forall x \in\left(b_{2}, b\right], \alpha_{1} \leq \alpha_{2} \Rightarrow \frac{f_{\alpha_{1}}(x)-c}{b-x} \leq \frac{f_{\alpha_{2}}(x)-c}{b-x}
\end{aligned}
$$

In the above definition, (23) means that when $y$ is neither very good nor very bad, by making $y$ a lot worse users can get $x$ a little better; (24) means that when $y$ is either very good or very bad, by making $y$ a lot worse users can get $x$ a little better; (25) means that when $y$ is very bad, the improvement in $x$ is increasing as $y$ gets worse (the more the value of $x$ approaching to the limit the harder the concession on $y$ ); and (26) means that when $y$ is the very good, the improvement in $x$ is decreasing as $y$ gets worse (the worse the value used to tradeoff the less its worth); (27) means that the less important $x$ is than $y$ the less concession at $y$ is needed to get $\mathrm{x}$ closer a little to its best value; and (28) means that the less important $x$ is than $y$ the less concession at $y$ is needed to get $\mathrm{x}$ better a little from its worst value.

The following theorem gives an adaptive tradeoff relation.

THEOREM 3. Suppose the domain of attribute $x$ is $[a, b]$ and the domain of attribute $y$ is $[c, d], \alpha \in[0,0.5)$ is a degree to which $x$ is more important than $y$. Then the relation, 
defined as

$$
y= \begin{cases}(d-c)\left(1-\left(\frac{x-a}{b-a}\right)^{2 \alpha}\right)+c & \text { if } x \in\left(x_{\text {bad }}, b\right] \\ \frac{y_{b a d}-y_{\text {good }}}{x_{\text {good }}-x_{\text {bad }}}\left(x-x_{\text {bad }}\right)+y_{\text {good }} & \text { if } x \in\left[x_{\text {good }}, x_{b a d}\right], \\ (d-c)\left(1-\frac{x-a}{b-a}\right)^{2 \alpha}+c & \text { if } x \in\left[a, x_{\text {good }}\right),\end{cases}
$$

is an adaptive tradeoff relation, where

$$
\begin{aligned}
& y_{\text {good }}=(d-c)\left(1-\left(\frac{x_{b a d}-a}{b-a}\right)^{2 \alpha}\right)+c, \\
& y_{b a d}=(d-c)\left(1-\frac{x_{\text {good }}-a}{b-a}\right)^{2 \alpha}+c .
\end{aligned}
$$

Now we apply the adaptive tradeoff relation (29) in our accommodation renting scenario. Let $k_{\text {good }}, k_{\text {bad }} \in[0,1]$ and $k_{\text {good }} \leq k_{\text {bad }}$. Then from (29), the adaptive tradeoff relation between rate $(r)$

$$
p=\left\{\begin{array}{c}
12\left(1-\left(\frac{1}{330} r\right)^{2 \alpha}\right) \\
\text { if } r \in\left(330 k_{b a d}, 330\right] \\
\frac{12\left(\left(1-k_{\text {good }}\right)^{2 \alpha}-\left(1-k_{\text {good }}^{2 \alpha}\right)\right)}{330\left(k_{\text {good }}-k_{\text {bad }}\right)}\left(r-330 k_{\text {bad }}\right)+12\left(1-k_{\text {bad }}^{2 \alpha}\right) \\
\text { if } r \in\left[330 k_{\text {good }}, 330 k_{\text {bad }}\right] \\
12\left(1-\frac{1}{330} r\right)^{2 \alpha} \\
\text { if } r \in\left[0,330 k_{\text {good }}\right)
\end{array}\right.
$$

and the adaptive tradeoff relation between rate $(r)$ and distance $(d)$ is as follows:

$$
d=\left\{\begin{array}{c}
30\left(1-\left(\frac{1}{330} r\right)^{2 \alpha}\right) \\
\text { if } r \in\left(330 k_{b a d}, 330\right], \\
\frac{30\left(\left(1-k_{\text {good }}\right)^{2 \alpha}-\left(1-k_{\text {good }}^{2 \alpha}\right)\right)}{330\left(k_{\text {good }}-k_{b a d}\right)}\left(r-330 k_{b a d}\right)+30\left(1-k_{b a d}^{2 \alpha}\right) \\
\text { if } r \in\left[330 k_{\text {good }}, 330 k_{b a d}\right], \\
30\left(1-\frac{1}{330} r\right)^{2 \alpha} \\
\text { if } r \in\left[0,330 k_{\text {good }}\right) .
\end{array}\right.
$$

In (32) and (33), if a user specified $\alpha=\frac{1}{4}, k_{\text {good }}=\frac{9}{25}$ and $k_{\text {bad }}=\frac{16}{25}$, then the adaptive tradeoff relation between rate and $\operatorname{period}(p)$ is as follows: ${ }^{6}$

$p= \begin{cases}12\left(1-\sqrt{\frac{1}{330} r}\right) & \text { if } r \in\left(\frac{16}{25} \times 330,330\right], \\ -\frac{6}{77}\left(r-\frac{16}{25} \times 330\right)+\frac{12}{5} & \text { if } r \in\left[\frac{9}{25} \times 330, \frac{16}{25} \times 330\right], \\ 12 \sqrt{1-\frac{1}{330} r} & \text { if } r \in\left[0, \frac{9}{25} \times 330\right)\end{cases}$

${ }^{6}$ There the $20 \%$ best values of $p$ are regarded as being very good and the $20 \%$ worst values of $y$ are regarded as being very bad. Thus, by (34) and (35), when $p_{\text {ver } y \text {-good }}=\frac{1}{5} \times 12$, $r_{\text {bad }}=\frac{16}{25} \times 330$, and when $p_{\text {very-bad }}=\frac{4}{5} \times 12, r_{\text {good }}=$ $\frac{9}{25} \times 330$.

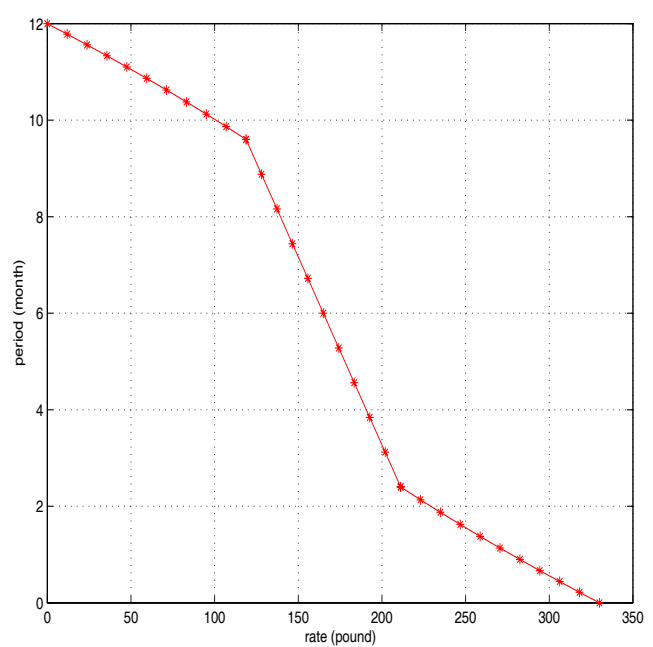

(a) Tradeoff between rate and period.

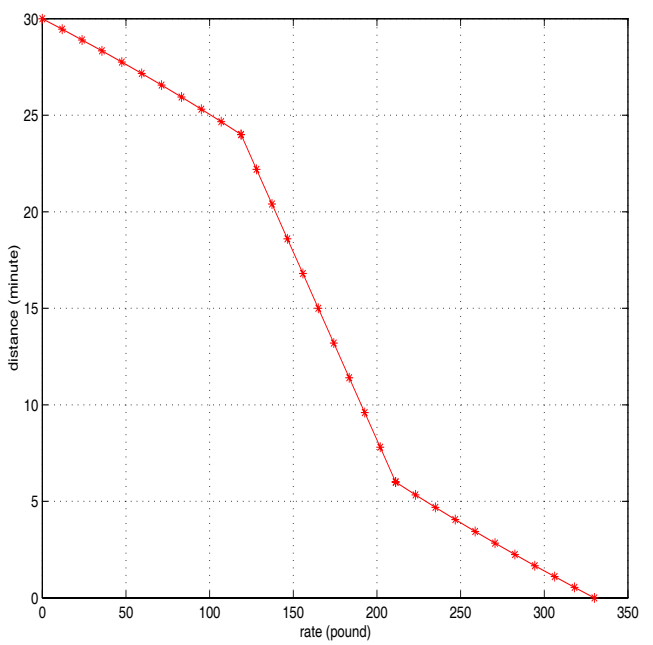

(b) Tradeoff between rate and distance.

Figure 4: Adaptive tradeoff relations.

and the adaptive tradeoff relation between rate $(r)$ and distance $(d)$ is as follows:

$d= \begin{cases}30\left(1-\sqrt{\frac{1}{330}}\right) & \text { if } r \in\left(\frac{16}{25} \times 330,330\right], \\ -\frac{15}{77}\left(r-\frac{16}{25} \times 330\right)+6 & \text { if } r \in\left[\frac{9}{25} \times 330, \frac{16}{25} \times 330\right], \\ 30 \sqrt{1-\frac{1}{330} r} & \text { if } r \in\left[0, \frac{9}{25} \times 330\right) .\end{cases}$

From the figures (Fig. 4) of these relations, it can be seen that:

- when period (or distance) is very good (i.e., less than $12 \times \frac{1}{5}$ for period or $30 \times \frac{1}{5}$ for distance), since it is viewed as more important than rate, the user is willing to make it a little worse in order to get rate a lot better; and

- when period (or distance) becomes not very good (i.e., more than $12 \times \frac{1}{5}$ for period), $30 \times \frac{1}{5}$ for distance) but rate is still not very good (i.e., less than $330 \times \frac{9}{25}$ ), the user will make period (or distance) a lot worse to get rate a little better, but this cannot go too far; thus 
- when period (or distance) becomes too bad (i.e., more than $12 \times \frac{4}{5}$ for period, $30 \times \frac{4}{5}$ for distance), the user will not make it a lot worse to get rate a little better, or say the attribute that is used to tradeoff rate is more important than rate again, and so the user makes it just a little worse to get rate a lot better.

Again, these points capture another viable way of trading off between attributes. For example, for an international $\mathrm{PhD}$ student who is seeking accommodation. Suppose she is preparing her $\mathrm{PhD}$ thesis and her husband wants to go back to their motherland as soon as possible. Thus, she wish to live as close to the university as possible so that she can get more time and finish her thesis as soon as possible. Accordingly, she regards the distance being important than more the rental rate when the distance is very good although the rental rate is very expensive. So, in this situation she is unwilling to increase distance a lot to get rate cheaper a little. However, when the distance becomes not very good she likes to increase distance a lot to decrease rate a little since in this situation she would like to take bus to her accommodation if she can save some money and taking bus will not significantly increase the time that she spends in the journey. Nevertheless, when the distance becomes too bad, even if she takes the bus it is not convenient for her and takes a lot time in the journey, and so she does not want to increase distance much to get rental rate better a little.

\section{GENERATION RULES}

From the analysis of the above three sections, we can see that tradeoff relations can be classified into three types: (1) a less important attribute is used to trade off another more important attribute, (2) a more important attribute is used to trade off another less important attribute, and (3) an attribute is used to trade off another attribute with the same importance. For the first type of tradeoff relations can be further classified into two subtypes: (1) the users do not want to stuck at the bottom line of the attribute that is used to trade off another attribute (i.e., a kind of quality tradeoff relations); and (2) the users do want to be stuck at the bottom line of the attribute that is used to trade off another attribute (i.e., impasse tradeoff relations). For the second type of tradeoff relations can be further classified into two subtypes: (1) the users care about all good values of the attribute that is used to trade off another attribute (i.e., another kind of quality tradeoff relations); and (2) the users do not care about not very good values and not very bad values of the attribute that is used to trade off another attribute (i.e., adaptive tradeoff relations). Thus, the generation rules that are used to choose concrete tradeoff relations are based on the following information: (1) which attributes the desired tradeoff is between, (2) which attribute is more important and how much it is more important, and (3) whether the tradeoff relations have the characteristics of impasse tradeoff relations or adaptive tradeoff relations.

Accordingly, for example, the generation rules for the accommodation renting scenario are as follows:

\footnotetext{
$R_{1}$ : IF the tradeoffs are between rate and period AND rate is more important than period AND do not want to be stuck at the bottom line of period
}

THEN the tradeoff relation is (8) with respect to relative importance degree $\alpha$ of rate against period

$R_{2}$ : IF the tradeoffs are between rate and period AND rate is more important than period AND want to be stuck at the bottom line of period until the rate becomes very good

THEN the tradeoff relation is (21) with respect to relative importance degree $\alpha$ of rate against period

$R_{3}$ : IF the tradeoffs are between rate and period AND rate is less important than period AND care about all good values of period

THEN the tradeoff relation is (8) with respect to relative importance degree $\alpha$ of rate against period

$R_{4}$ : IF the tradeoffs are between rate and period AND rate is less important than period AND do not care about not very good and not very bad values of period

THEN the tradeoff relation is (32) with respect to relative importance degree $\alpha$ of rate against period

$R_{5}:$ IF the tradeoffs are between rate and period AND rate is equally important as period

THEN the tradeoff relation is (8) with respect to relative importance degree $\alpha=0.5$

$R_{6}:$ IF the tradeoffs are between rate and distance AND rate is more important than distance AND do not want to be stuck at the bottom line of distance

THEN the tradeoff relation is (9) with respect to relative importance degree $\alpha$ of rate against distance

$R_{7}:$ IF the tradeoffs are between rate and distance AND rate is more important than distance AND want to be stuck at the bottom line of distance until the rate becomes very good

THEN the tradeoff relation is (22) with respect to relative importance degree $\alpha$ of rate against distance

$R_{8}:$ IF the tradeoffs are between rate and distance AND rate is less important than distance AND care about all good values of distance

THEN the tradeoff relation is (9) with respect to relative importance degree $\alpha$ of rate against distance

$R_{9}:$ IF the tradeoffs are between rate and distance AND rate is less important than period AND do not care about not very good and not very bad values of distance

THEN the tradeoff relation is (33) with respect to relative importance degree $\alpha$ of rate against distance

$R_{10}:$ IF the tradeoffs are between rate and distance AND rate is equally important as distance

THEN the tradeoff relation is (9) with respect to relative importance degree $\alpha=0.5$

\section{RELATED WORK}

The background of our work is knowledge acquisition and preference elicitation. There are many methods for acquiring knowledge from humans for knowledge-based system in general (see [17] for an overview). However, usually they 
do not aim at eliciting user preferences for decision making. Fortunately, there are also some methods for preference elicitation for decision making problems. The most common ones elicit a user's preferences by means of utility functions. For instance, (1) gambling elicitation techniques [23] in which the utility of one outcome is ascertained by comparing it to a lottery involving two others; and (2) visual exploration and incremental utility elicitation [3] in which suggestions are made visually to a human user based on an incomplete model of their utility function and the model is updated based on the feedback from the user. However, the workload that users use these methods to elicit preferences is very high. In order to address the limitation, some special ways to acquire preferences from users have been proposed. For example, (1) the case based preference elicitation [12, $14,13]$ that requires a user to provide partial information about their preference and then constructs the whole preference structure via choosing the most similar one in the case base of preference structure; and (2) preference elicitation via theory refinement $[10,27,15]$ that starts with approximate and incomplete domain knowledge and then corrects inaccuracies and incompleteness by training on examples (i.e., knowledge-based artificial neural networks are used to learn user preferences).

While a procedure of negotiation can be viewed as the one of dynamic decision making over time, our tradeoff preference elicitation for negotiation is different from preference elicitation for decision making in general. In the area of preference elicitation for decision making problems, the term preference refers to ordering on a set of alternatives and its focus is on eliciting the ordering; little work is on eliciting alternatives. In this paper, our focus is on eliciting tradeoff alternatives. As to the problem of eliciting ordering on a set of tradeoff alternatives (i.e., the preference on tradeoff alternative), we just use a method of direct rating in order to highlight the elicitation of tradeoff alternatives. In future work, we believe it is worth merging more elaborate methods for preference elicitation into our methods for eliciting tradeoff alternatives.

While existing techniques for constraint elicitation have shown some success in particular contexts, they are not easily able to handle our tradeoff acquisition problem. This is true even though in our model the users' tradeoff preferences are represented by fuzzy constraint. Firstly, a number of methods have been proposed to acquire crisp constraints. For example, [24] presents an approach to interactive constraint acquisition based on the techniques of Mitchell's List-ThenEliminate method [22]. In this approach, there is a "hypothesis space" of constraints over which a general-to-specific ordering is known (or is implicit in the hypothesis space representation). Then the user's examples (where a constraint should be satisfied or violated) are used to eliminate hypotheses that are inconsistent with the examples. In this procedure, a strategy is also employed to minimise the dialog length between the user and the computer. However, this method is only applicable to crisp constraints. Secondly, Biso et al. used neural networks to attack the problem of soft constraint acquisition [2] (fuzzy constraints are a special case of soft constraints). However, their method is used to learn the solution rating function given a fixed constraint graph and some examples of solution ratings. Thus, their method assumes the constraints are already available, and what their method learns is how to rate a solution according to the available constraints. It does not resolve the problem of how to acquire soft constraints themselves.

Perhaps the most related work to ours is that of [9] that aims to acquire tradeoffs in a user's preferences. However, there are a number of important differences. First, [9] uses heuristic strategies to generate various tradeoffs and then asks the user to confirm whether they are acceptable (their aim is to improve the efficiency of the acquisition process). This approach may cause some tradeoffs to be missed in complex multi-dimensional spaces and so we focus on a more systematic exploration of the space based on knowledge about the types of tradeoff relation. Second, the method in [9] is not designed for negotiation problems, and their example scenario, the N-Queens problem, is much more objective than that typically used in negotiations. Third, in [9] tradeoffs are crisp (i.e., the user regards each tradeoff as an equal alternative), but in this paper tradeoffs are fuzzy (i.e., for different alternative tradeoffs the user has different satisfaction degrees, or simply the user has a preference on tradeoff alternatives). Fourth, [9] does not identify various types of tradeoff relation, but we do and moreover, we gave an instance for each type. This significantly reduces the users' burden.

Finally, in the research area of agent-based automated negotiation little work has addressed the problem of preference acquisition from human users.

- The work [18] provides a method for one agent to elicit preferences from another agent in combinatorial auction settings. However, this work assumes that the user has already imparted their preferences into the negotiating agent (the aim of our work presented in this paper) and the aim of their elicitation process is to prune the auctioneer agent's search space.

- In another work [4], negotiation and preference elicitation is linked. However, the work is different from ours in the following aspects: (1) Its preference elicitation does not refer to user preference elicitation. Actually, it refers to a program (an automated resource manager) that elicits the preferences on resources from other programs (workload managers). Rather, the aim of our work in this paper is to elicit tradeoff preferences from users. (2) The process of its preference elicitation is viewed as a cooperative negotiation between an automated resource manager and workload managers. In other words, the motivation of its elicitation is not for a program to negotiate faithfully on behalf of its human owner (the motivation of our work in this paper). In fact, its motivation is to find near-optimal allocations of resources to distinct computing elements in large, distributed computing systems.

- In our work [5], the most valuable contribution is that it can acquire seller's rewards and restrictions to products, but user tradeoff preference elicitation is not involved there. Acquiring buyer's tradeoffs on negotiation issues is the focus of this paper. So, they are complementary to each other. 
- The work in [11] employs an evolution algorithm to address the problem of preference elicitation in form of utility functions. However, there term preference is general rather than tradeoff preference. As a result, it is not distinguished from the research area of general user preference elicitation.

\section{CONCLUSIONS AND FUTURE WORK}

This paper highlights the importance of knowledge acquisition for negotiation agents. In particular, we develop a knowledge-based method for acquiring user tradeoff preferences and identify three common types of tradeoff relations. This is very important because tradeoffs are one of the fundamental things in the principled negotiation approach [8]. Moreover, our negotiation model [21] partially realised the principled negotiation approach. The approach is recognised as one of the best approaches for manual bilateral negotiations and it has been shown to be applicable to many domains. So, although we use an accommodation renting domain as the example, our methods are also generally applicable.

Motivating more work is one of the important aims of this paper. We think the following issues are worth further studying:

- Other types of knowledge used in negotiation models. For the principled negotiation approach [8], there are three classes of knowledge used in negotiation: (1) knowledge for interest-based negotiation, (2) knowledge for making tradeoffs, and (3) knowledge for argumentation. This paper focuses on eliciting the second class; the issues of eliciting the other two need to be studied. More generally, work is needed to determine if there are knowledge requirements that are more common to many negotiation algorithms and, if so, what knowledge acquisition methods can be applied to these broad classes of requirements.

- Other methods of user tradeoff preference elicitation. The exhaustive method proposed in another paper [20] can be viewed as a base line of user tradeoff preference elicitation; its high elicitation cost can motivate a lot of further work. The knowledge-based method developed in this paper is an example that is motivated by the high elicitation cost of the first method. Like what has been done in the area of general preference elicitation, many heuristic methods (e.g., neural networks, case based reasoning and inductive learning) can be used for reducing the elicitation workload of user tradeoff preferences.

- Other types of tradeoff: (1) beyond the trading off of one issue against another; (2) beyond the linearity of preferences (e.g., a user may prefer tradeoff alternative $a$ to tradeoff alternative $b$, and tradeoff alternative $a$ to tradeoff alternative $c$, but have no preference between $b$ and $c$ ); and (3) beyond the crisp curves of tradeoff relations (e.g., two alternatives: (i) if rate is about 260 pounds then the distance is about 20 minutes walking; and (ii) if rate is about 270 pounds then the distance is about 15 minutes walking).
- Other types of negotiation model. Besides constraintbased negotiation models, other two common classes of negotiation models are utility-based ones and reasoningbased ones. The utility acquisition have been revisited and refined over the past 50 years, and the main results of this research effort are now well established. As to reasoning based models, knowledge used for reasonings could be modelled as Bayesian networks, fuzzy rules, or cases; there also exist well-established methods for acquiring these forms of knowledge from data or from human users. These existing methods can be applied for acquiring knowledge for reasoning-based negotiation systems.

\section{Acknowledgements}

This work is supported by Hewlett Packard Research Labs. Besides, authors are very much obliged to Partha S. Dutta for his proof-reading.

\section{REFERENCES}

[1] M. Ai. Handbook of Negotiation and Contract Signing. Internal Mongolia Culture Publishing House, 2002. In Chinese.

[2] A. Biso, F. Rossi, and A. Sperduti. Experimental results on learning soft constraints. In Proceedings of the International Conference on Principles of Knowledge Representation and Reasoning, 2000.

[3] J. Blythe. Visual exploration and incremental utility elicitation. In Proceedings of the Eighteenth National Conference on Artificial Intelligence, pages 526-532, 2002 .

[4] C. Boutilier, R. Das, J.O. Kephart, G. Tesauro, and W.E. Walsh. Cooperative negotiation in autonomic systems using incremental utility elicitation. In Proceedings of the nineteenth International Conference on Uncertainty in Artificial Intelligence, Acapulco, Mexico, August 2003.

[5] J.J. Castro-Schez, N.R. Jennings, X. Luo, and N.R. Shadbolt. Acquiring domain knowledge for negotiating agents: A case of study. International Journal of Human Computer Studies, 2003. To Appear.

[6] M. Dastani, N. Jacobs, C.M. Jonker, and J. Treur. Modeling user preferences and mediating agents in electronic commerce. In F. Dignum and C. Sierra, editors, Agent Mediated Electronic Commerce: The European AgentLink Perspective, volume 1991 of Lecture Notes in Artificial Intelligence, pages 163-193. Springer, 2001.

[7] P. Faratin, C. Sierra, and N.R. Jennings. Using similarity criteria to make issue tradeoffs in automated negotiations. Artificial Intelligence, 142(2):205-237, 2002.

[8] R. Fisher and W. Ury. Getting to yes: Negotiating an agreement without giving in. Random House Business Books, 1981.

[9] E.C. Freuder and B. O'Sullivan. Modeling and generating tradeoffs for constraint-based configuration. In Proceedings of IJCAI-01 Workshop on Configuration, 2001. 
[10] B. Geisler, V. Ha, and P. Haddawy. User modeling via theory refinemen. In Proceedings of the International Conference on Intelligent User Interface, 2001.

[11] Y. Guo, J.P. Muller, and C. Weinhardt. Learning user prferences for multi-attribute negotiation: An evolutionary approach. In J. Muller, V. Marik, and M. Pechoucek, editors, Multi-Agent Syetems and Applications III, volume 2691 of Lecture Notes in Artificial Intelligence, pages 303-313. Springer, 2003.

[12] V. Ha and P. Haddawy. Toward case-based preference elicitation: Similarity measures on preference structures. In Proceedings of the Fourteenth International Conference on Uncertainty in Artificial Intelligence, pages 193-201, 1998.

[13] V. Ha and P. Haddawy. Similarity of personal preferences: Theoretical foundations and empirical analysis. Artificial Intelligence, 146(2):149-173, 2003.

[14] V. Ha, P. Haddawy, and J. Miyamoto. Similarity measures on preference structure, part ii: Utility functions. In Proceedings of the Seventeenth International Conference on Uncertainty in Artificial Intelligence, pages 186-193, 2001.

[15] P. Haddawy, V. Hu, B. Geisler, and J. Miyamoto. Preference elicitation via theory refinement. Journal of Machine Learning Research, 2003. to appear.

[16] M. He, N.R. Jennings, and H.F. Leung. On agent-mediated electronic commerce. IEEE Transactions on Knowledge and Data Engineering, 15(4):985-1003, 2003.

[17] R.R. Hoffman and N.R. Shadbolt. Eliciting knowledge from experts: A methodological analysis. Organizational and Human Decision Process, 62(2):129-158, 1995.

[18] B. Hudson and T. Sandholm. Effectiveness of preference elicitation in combinatorial auctions. In Proceedings of Agent-Mediated Electronic Commerce (AMEC) workshop at AAMAS-02, 2002.

[19] A.R. Lomuscio, M. Wooldridge, and N.R. Jennings. A classification scheme for negotiation in electronic commerce. International Journal of Decision and Negotiation, 12(1):31-56, 2003.

[20] X. Luo, N.R. Jennings, and N. Shadbolt. Acquiring tradeoff preferences for automated negotiations: A case study. In Proceedings of the 5th International Workshop on Agent-Mediated E-Commerce, Melbourne, Australia, 2003.

[21] X. Luo, N.R. Jennings, N. Shadbolt, H.F. Leung, and J.H.M. Lee. A fuzzy constraint based model for bilateral, multi-issue negotiation in semi-competitive environments. Artificial Intelligence, 148(1-2), 2003.

[22] T. Mitchell. Concept learning and the general-to-specific ordering. In Machine Learning, chapter 2, pages 20-51. McGraw Hill, 1997.
[23] J. Von Neumann and O. Morgenstern. Theory of Games and Economic Behaviour. Princeton University Press, 1944.

[24] S. O'Connell, B. O'Sullivan, and E.C. Freuder. Strategies for interactive constraint acquisition. In Proceedings of CP-02 Workshop on User-Interaction in Constraint Satisfaction, New York, 2002.

[25] D. Pruitt. Negotiation Behavior. Academic Press, 1981.

[26] H. Raiffa. The Art and Science of Negotiation. Harvard University Press, Cambridge, USA, 1982.

[27] A. Reistificar, P. Haddawy, V. Ha, and J. Miyamoto. Eliciting utilities by refining theories of monotonicity and risk. In Working Notes of the AAAI'2002 Workshop on Preferences in AI and CP: Symbolic Approache, 2002.

[28] G. Schreiber and H. Akkermans Knowledge-Model Language. In Knowledge Engineering and Management: The CommonKADS Methodology, pages 403-418. The MIT Press, 2000.

[29] P.T. Steel and T. Beasor. Business negotiation: A practical workbook. Gower Publishing Limited, 1999.

[30] I. Unt. Negotiation Without A Loser. Copenhagen Business School, 1999.

[31] R.J. Volkema. The negotiation toolkit: How to get exactly what you want in any business or personal situation. Amacom, 1999. 